\title{
Crystalline Wax Esters Regulate the Evaporation Resistance of Tear Film Lipid Layers Associated with Dry Eye Syndrome
}

\section{Paananen, Riku O.}

2019-07-18

Paananen, R O , Javanainen, M , Holopainen, J M \& Vattulainen , I 2019 , ' Crystalline Wax Esters Regulate the Evaporation Resistance of Tear Film Lipid Layers Associated with Dry Eye Syndrome ' , Journal of Physical Chemistry Letters , vol. 10 , no. 14 , pp. 3893-3898 . https://doi.org/10.1021/acs.jpclett.9b01187

http://hdl.handle.net/10138/306068

https://doi.org/10.1021/acs.jpclett.9b01187

publishedVersion

Downloaded from Helda, University of Helsinki institutional repository.

This is an electronic reprint of the original article.

This reprint may differ from the original in pagination and typographic detail.

Please cite the original version. 


\title{
Crystalline Wax Esters Regulate the Evaporation Resistance of Tear Film Lipid Layers Associated with Dry Eye Syndrome
}

\author{
Riku O. Paananen, ${ }^{*}, \dagger$ Matti Javanainen, ${ }^{\perp, \S, \|_{\odot}}$ Juha M. Holopainen, ${ }^{\dagger, \mathbb{I}}$ and Ilpo Vattulainen ${ }^{*},\|\|,, \nabla_{\odot}$ \\ ${ }^{\dagger}$ Helsinki Eye Lab, Department of Ophthalmology, University of Helsinki and Helsinki University Hospital, FI-00014 Helsinki, \\ Finland \\ ${ }^{\ddagger} J$. Heyrovský Institute of Physical Chemistry, Czech Academy of Sciences, CZ-18223 Prague 8, Czech Republic \\ ${ }^{\perp}$ Institute of Organic Chemistry and Biochemistry, Czech Academy of Sciences, CZ-16610 Prague 6, Czech Republic \\ ${ }^{\S}$ Department of Physics, University of Helsinki, FI-00014 Helsinki, Finland \\ "Computational Physics Laboratory, Tampere University, FI-33100 Tampere, Finland \\ ${ }^{\nabla}$ MEMPHYS-Centre for Biomembrane Physics
}

Supporting Information

\begin{abstract}
Dry eye syndrome (DES), one of the most common ophthalmological diseases, is typically caused by excessive evaporation of tear fluid from the ocular surface. Excessive evaporation is linked to impaired function of the tear film lipid layer (TFLL) that covers the aqueous tear film. The principles of the evaporation resistance of the TFLL have remained unknown, however. We combined atomistic simulations with Brewster angle microscopy and surface potential experiments to explore the organization and evaporation resistance of films composed of wax esters, one of the main components of the TFLL. The results provide evidence that the evaporation resistance of the TFLL is based on crystalline-state layers of wax esters and that the evaporation rate is determined by defects in the TFLL and its coverage on the ocular surface. On the basis of the results, uncovering the nonequilibrium spreading and crystallization of TFLL films has potential to reveal new means of treating DES.
\end{abstract}

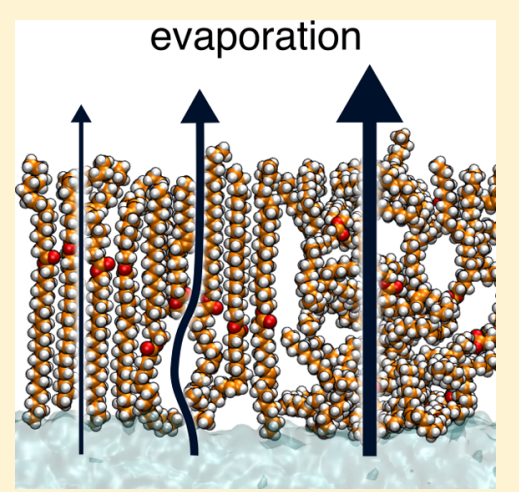

$\mathrm{D}$ ry eye syndrome (DES) is a disease associated with a chronic lack of sufficient lubrication on the eye surface. It is characterized by irritation, watering of eyes, blurred vision, and a sensation of pain. In the most severe cases DES may result in ocular inflammation, ulcers, and even permanent scarring. Given these issues, DES poses a serious health problem. In the United States alone, tens of millions of people suffer from DES, ${ }^{1}$ and worldwide it affects up to one-third of the world's population. ${ }^{1}$

The issues of DES focus on the eye surface that is covered by a tear film, which is divided into a muco-aqueous gel layer and a tear film lipid layer (TFLL). People without dry eyes have TFLLs that retard the evaporation of aqueous tear fluid, ${ }^{2}$ thus maintaining moisture on the ocular surface. People who have dry eyes, however, suffer from conditions where the ocular surface is no longer naturally moist. A key factor, as a common cause of DES, is excess evaporation of tear fluid due to a dysfunctional TFLL. ${ }^{3}$

However, the mechanism by which the TFLL of non-DES subjects is able to prevent evaporation has remained a mystery. Unlike monolayers of polar lipids composed of fatty acids and fatty alcohols that effectively retard evaporation of water, ${ }^{4}$ the TFLL is mainly composed of nonpolar cholesteryl esters (CEs) and wax esters (WEs). ${ }^{5-7}$ Despite their nonpolar nature, recent studies on model lipid monolayers have shown that WE films can effectively retard the evaporation of water ${ }^{8,9}$ and that the properties slowing down evaporation are related to the crystalline structure of WE films. ${ }^{9,10}$ It is tempting to ask whether crystalline WEs could also be responsible for the evaporation resistance of the TFLL, because the TFLL is in a semicrystalline state. ${ }^{11-13}$ Clinical data support this view, because lipidomic studies have revealed concentrations of specific wax esters of DES patients to be reduced compared to those of people without DES. ${ }^{14}$ Meanwhile, the idea that the behavior of model lipid monolayers could be used to understand TFLL function is not obvious, because TFLLs are about $10-100 \mathrm{~nm}$ thick and characterized by a complex molecular composition. ${ }^{5-7}$ However, a key fact is that the bulk of the TFLL (under the water-TFLL interface) is rich in WEs whose ability to prevent evaporation in TFLL environments has not been explored. If WEs are the primary factor in preventing evaporation, then how do they do it, and how could this information be utilized in developing new treatments for DES?

To tackle this, we developed an all-atom molecular dynamics (MD) model for WEs, the aim being to correctly describe the crystalline order and the spreading properties of WE films.

Received: April 25, 2019

Accepted: June 25, 2019

Published: June 25, 2019 
A

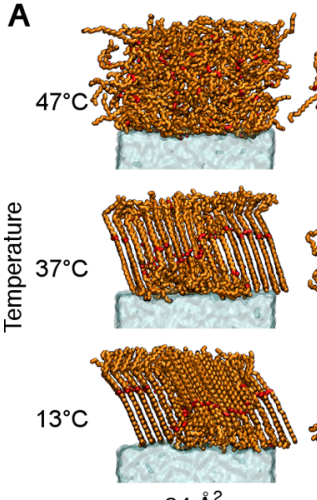

$24 \AA^{2}$
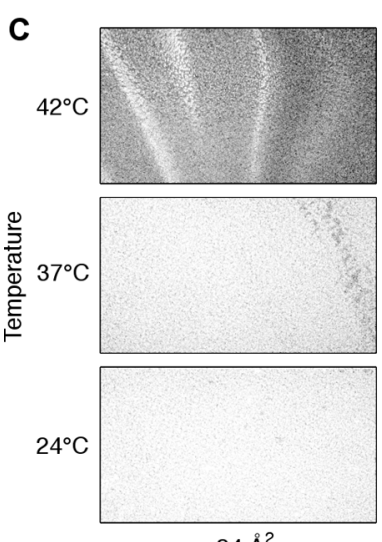

$24 \AA^{2}$
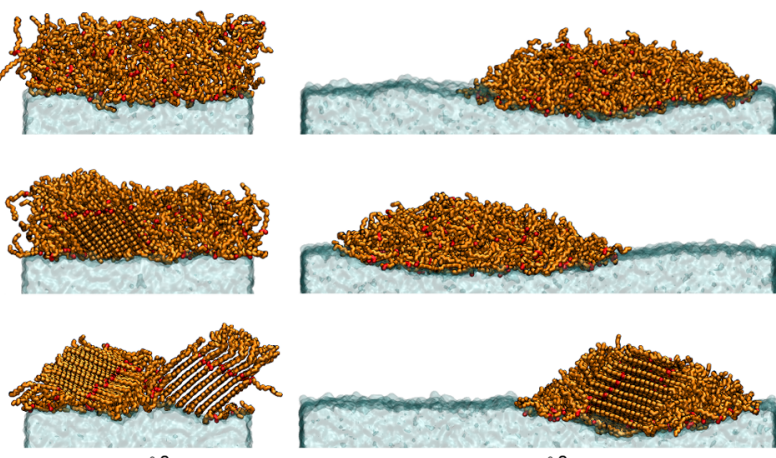

$35 \AA^{2}$ $\operatorname{MMA}\left(\AA^{2} /\right.$ molecule $)$
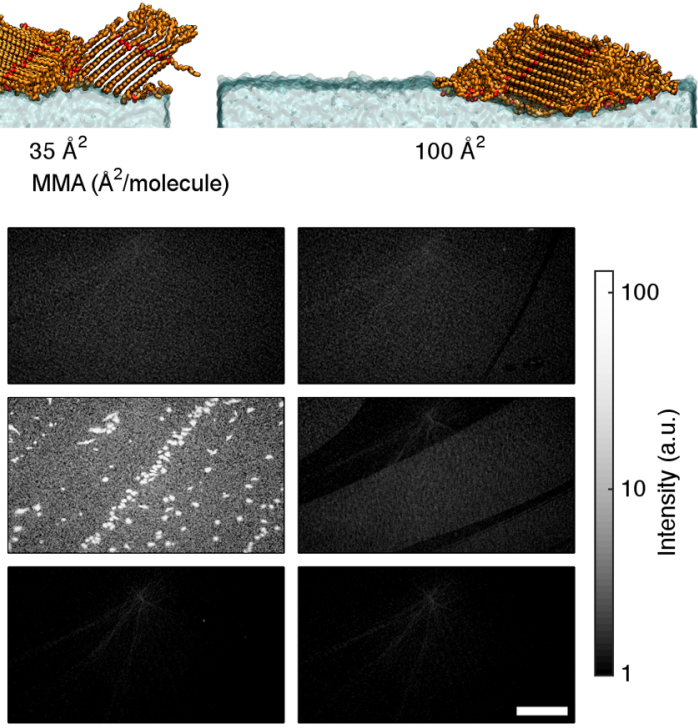

$40 \AA^{2}$ $\operatorname{MMA}\left(\AA^{2} /\right.$ molecule $)$
$100 \AA^{2}$

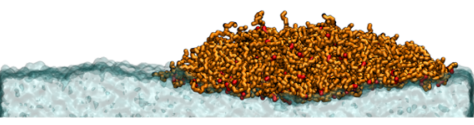

B
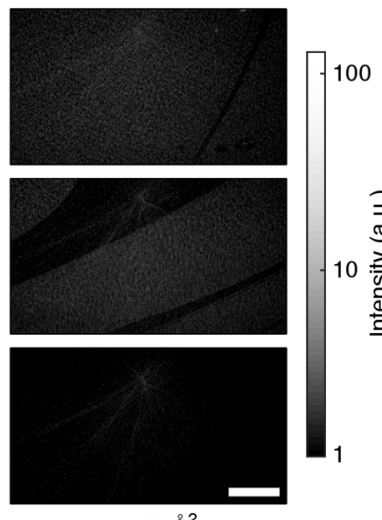

$80 \AA^{2}$

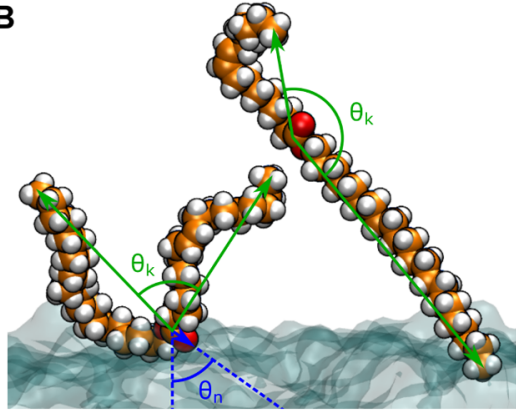

D

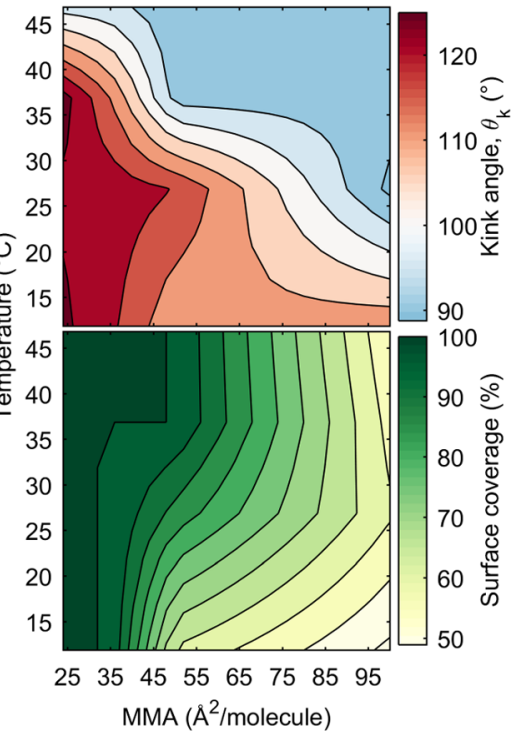

Figure 1. Organization of behenyl oleate (BO) films. (A) Snapshots of simulated BO films after equilibration. Color code: red, ester group; orange, hydrocarbon chains; and blue, water. Hydrogens are not shown for clarity. (B) Two main BO conformations and the definition of the angles used in the analysis. Color code: red, oxygen; orange, carbon; and white, hydrogen. (C) Brewster angle microscope images of BO films. Note the logarithmic intensity scale. Scale bar is $500 \mu \mathrm{m}$. (D) Mean kink angle and proportion of the water surface covered by BO computed from the MD simulations.

Molecular simulations have established themselves as one of the excellent methods for studying the properties of biomembranes. ${ }^{15}$ In this work, the simulation model used in our studies is based on the widely used OPLS ${ }^{16}$ force field and the recently developed models for hydrocarbons ${ }^{17}$ and water. ${ }^{18}$ This combination gives a valid description of both the WE bulk properties (see the Supporting Information) and the surface tension of water, ${ }^{19}$ making it optimal for WE layer simulations. The simulations were carried out systematically over an extensive range of phase space and were complemented by surface potential and Brewster angle microscopy (BAM) measurements applied to model TFLL (Langmuir) monolayers composed of WEs. The simulation results were consistent with experimental findings and successfully explained experimental results on a molecular level. Altogether, the simulations and experimental results provide compelling evidence that in TFLLs crystalline-state WE regions play a decisive role in preventing or retarding the evaporation of tear fluid. Interestingly, in eye drops currently used to treat DES, WEs are not involved. The present results therefore suggest a new approach for treating this disease.

We chose behenyl oleate (BO; Figure $1 \mathrm{~B}$ ) as the wax ester to be investigated, because from the commercially available WEs, BO is the closest match to the most abundant tear film WEs. The melting point ${ }^{9}$ of $\mathrm{BO}$ is $38^{\circ} \mathrm{C}$; therefore, $\mathrm{BO}$ is in a crystalline state at the ocular surface, whose temperature is around $33-35{ }^{\circ} \mathrm{C}$. We study these WE layers at numerous packing conditions characterized by the mean molecular area (MMA) along the air-water interface to describe varying surface pressure during eye blinking. After validating the WE simulation model (see Figure S2), we studied the phase behavior of BO films at the air-water interface by combining MD simulations (Figure 1A) with BAM (Figure 1C). Here, BAM delineates the macroscale appearance of the film, while MD simulations reveal their nanoscale organization.

The studies demonstrated three different types of organization depending on the temperature of the system. Here, we first consider the high-temperature case. At $42{ }^{\circ} \mathrm{C}$, BAM measurements showed that $\mathrm{BO}$ forms a film, which resembles a condensed monolayer on a microscopic scale (Figure 1C). As MMA was decreased, full surface coverage (Figure 1C) was observed at MMA $\leq 60 \AA^{2} /$ molecule, where the film formed an overlying bulk-like layer (Figure $1 \mathrm{C}$ ). As seen from the BAM images (Figure 1C), this overlying layer at low MMA aggregated into droplets instead of forming a stable duplex layer. For comparison, MD simulations indicated that at low MMA the film is disordered (Figure 1A), and individual WEs were characterized by an average kink angle (defined as the angle between the carbonyl carbon and two methyl carbons, see Figure $1 \mathrm{~B}$ ) of $90-105^{\circ}$, matching a kink angle of $101^{\circ}$ observed in bulk BO above its melting point (Figure 1D). Here, $\sim 50 \%$ of the ester groups were oriented toward the 
water phase. While at large MMA the disordered layer did not cover the full surface (Figure 1A,D), for MMA less than $~ 50-$ $60 \AA^{2} /$ molecule, the simulations highlighted uniform but thicker droplet-like layers along the surface, in line with the BAM data.

Second, at $37{ }^{\circ} \mathrm{C}$, slightly below the melting point of BO, simulations highlighted spreading (similar to behavior observed at higher temperatures) (Figure 1A,D), but the collapse took place by formation of crystalline regions of molecular thickness. Within the crystalline regions, molecules adopted extended conformations as shown by the kink angle of $\sim 125^{\circ}$ (Figure 1D). This feature was observed in BAM images (Figure 1C) as the appearance of thick, solid crystals with uniform intensity.

Third, at room temperature $\left(24^{\circ} \mathrm{C}\right)$, well below the melting point of $\mathrm{BO}$, simulations revealed the kink angle to be high (Figure 1D), and the film was completely crystalline and did not spread, leaving the water surface exposed even for MMA as low as $\sim 35-40 \AA^{2} /$ molecule. The same behavior was observed in the BAM images (Figure 1C).

As shown in Figure 1, spreading occurred at temperatures below the melting temperature. However, because of the low equilibrium spreading pressure of $\mathrm{BO}\left(\sim 1 \mathrm{mN} \mathrm{m}^{-1}\right)$, spreading from solid crystals begins just a few degrees before the melting point, as described earlier for behenyl palmitoleate. ${ }^{10}$ The results also show that the spreading film is not strictly monomolecular, and molecular orientation in the film is only weakly affected by the presence of the water surface.

Equilibrium between solid crystals and a disordered thin film appears to be the key to the evaporation retarding properties of WE films, because WEs slow down evaporation only in the temperature range of less than $10{ }^{\circ} \mathrm{C}$ below their melting point. ${ }^{9,10}$ This is likely due to spreading, which promotes twodimensional crystallization at the water surface over formation of three-dimensional crystals and aggregation. This leads to high surface coverage of the solid crystal phase and effective reduction of evaporation.

Because it is currently impractical to perform simulations that would be long enough to render spontaneous crystal nucleation possible, an initial nucleus is instead required (see the Supporting Information). Obviously, the molecular conformation within the initial nucleus will have an impact on the final crystal structure. In the current study, the initial conformation of the crystal nucleus used in the simulations was based on reported structural data on saturated wax esters. ${ }^{20-22}$ However, it is possible that crystals forming at the air-water interface may differ from those in the bulk.

Because of this limitation, we looked for additional confirmation that the nanoscale organization revealed by the MD simulations is accurate. To this end, we compared the surface potential calculated from equilibrated $\mathrm{BO}$ monolayer simulations with experimentally measured data (Figure 2A) and found good qualitative agreement. In experiments at 42 ${ }^{\circ} \mathrm{C}$, above the melting point of $\mathrm{BO}$, surface potential increased during compression until a limiting area of $\sim 60 \AA^{2} /$ molecule was reached. For smaller MMA, a plateau was observed at $\sim 235 \mathrm{mV}$ (corresponding to an average vertical dipole moment of $375 \mathrm{mD}$ ). This can be attributed to the collapse of excess BO over the monolayer (Figure 1). The low vertical molecular dipole moment compared to expanded monolayers of ethyl and butyl esters (approximately $540 \mathrm{mD})^{23}$ also supports the view of a disordered layer organization, with ester groups only weakly oriented toward the water phase as
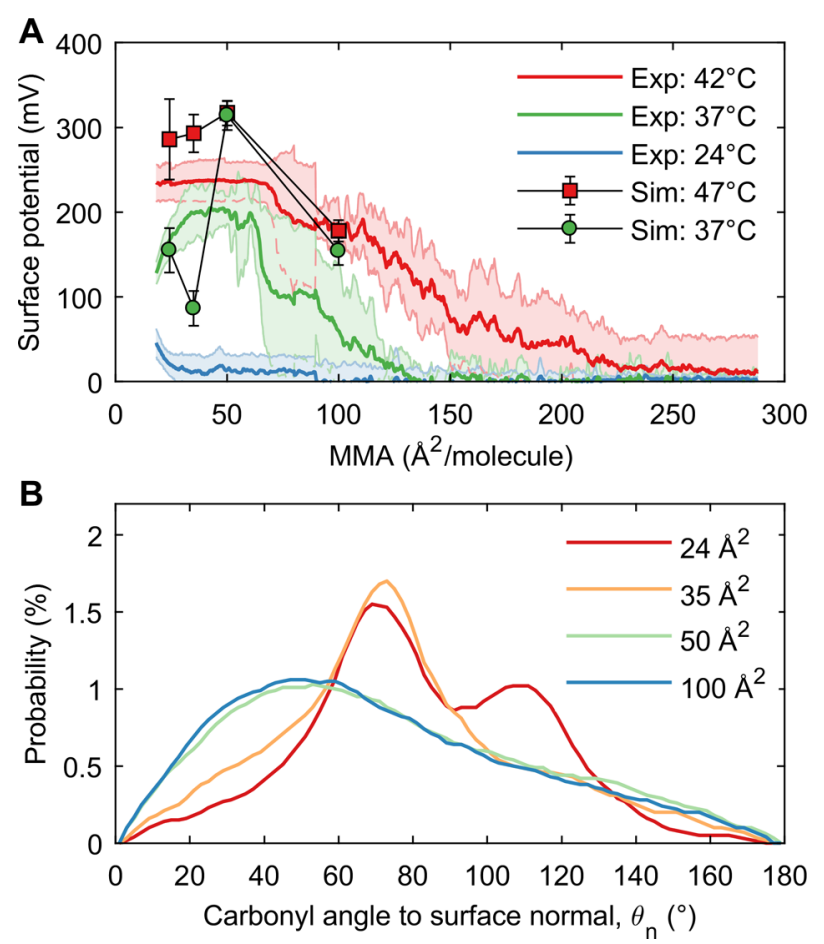

Figure 2. (A) Surface potential of behenyl oleate films. Solid lines: experimentally determined surface potential, where shaded regions depict standard deviation calculated from at least 4 compressionexpansion measurements. Markers: simulation data. Markers and lines: surface potential from MD simulations. Error bars were determined using block averaging. (B) Distribution of the angle between the $\mathrm{BO}$ carbonyl bond and surface normal $\left(\theta_{\mathrm{n}}\right.$, see Figure 1B) calculated from $\mathrm{MD}$ simulations at $37^{\circ} \mathrm{C}$.

described above. Simulations above the melting point are qualitatively in agreement with this picture.

At $37{ }^{\circ} \mathrm{C}$, right under the melting temperature, surface potential again increased during compression; however, at $\sim 40$ $\AA^{2} /$ molecule the surface potential collapsed. Simulations described a similar collapse. This decrease is associated with the reorientation of WEs during the formation of the crystal phase: the ester dipoles undergo a change where they become largely parallel to the air-water interface (Figure 2B).

In experiments at room temperature $\left(24^{\circ} \mathrm{C}\right)$, no spreading occurred. The individual floating solid crystals were sensed by a surface potential probe only if they were strongly packed (Figure 1B).

Currently available experimental methods can only determine the average evaporation resistance of WE films, but $\mathrm{MD}$ simulations are also able to quantify the evaporation resistance of different film regions. In the evaporation simulations, we calculated the potential of mean force (PMF) using umbrella sampling and extracted the diffusion coefficients from the mean-squared displacement (MSD). The MMA was set to 24 $\AA^{2} /$ molecule, and the temperature was chosen as $27^{\circ} \mathrm{C}$. The evaporation resistance of $\mathrm{BO}$ films was determined using the inhomogeneous solubility-diffusion model ${ }^{24}$ for three different cases: a disordered film, a perfectly crystalline film with no defects, and a crystalline film with a boundary between two crystallites. For all three cases, the density, diffusion coefficient, PMF, and permeation resistance profiles are shown in Figure 3A-D.

Within a disordered BO film, the diffusion coefficient and free energy were almost constant, as expected for a 

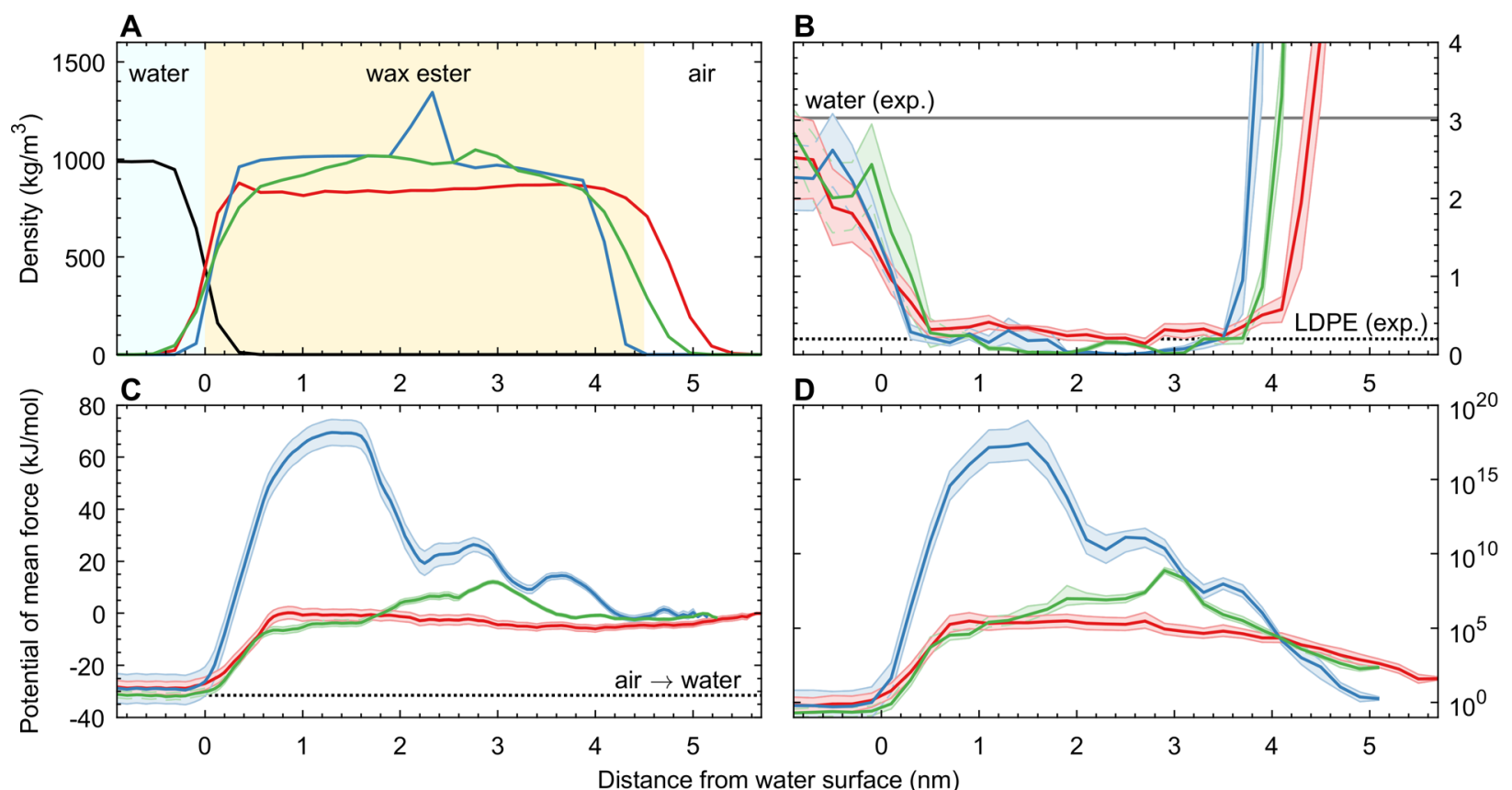

Figure 3. Results of the permeation simulations of $\mathrm{BO}$ films at a mean molecular area of $24 \AA^{2} /$ molecule at $27^{\circ} \mathrm{C}$. In all panels the color coding is as follows: red, disordered $\mathrm{BO}$ film; blue, crystalline BO film; and green, crystalline BO film with a domain boundary. Shaded regions depict error estimates. (A) Density profiles of the interface, with black describing average water density. (B) Diffusion coefficient profiles. For comparison, horizontal lines depict diffusion coefficients in water ${ }^{40}$ (full line) and amorphous low-density polyethylene (LDPE) ${ }^{28}$ (dotted line). (C) Freeenergy profiles. Dotted line depicts the hydration free energy (see the Supporting Information). (D) Permeation resistance profiles. Note the logarithmic $y$-axis.

homogeneous film. Results show no additional evaporation resistance for the surface film at the $\mathrm{BO}$-water interface. The diffusion coefficient of water within the disordered $\mathrm{BO}$ film was observed to be $(0.28 \pm 0.02) \times 10^{-5} \mathrm{~cm}^{2} \mathrm{~s}^{-1}$ (Figure 3B), and a change in free energy was identified as $(-2.2 \pm 0.6) \mathrm{kJ}$ $\mathrm{mol}^{-1}$ with respect to the gas phase (Figure $3 \mathrm{C}$ ), resulting in a permeation resistance of approximately $(1.9 \pm 1.2) \times 10^{5} \mathrm{~s}$ $\mathrm{cm}^{-2}$ (Figure 3D). No experimental values were found in the literature for $\mathrm{BO}$, but for liquid hexadecane at $35{ }^{\circ} \mathrm{C}$, a diffusion coefficient of $4.95 \times 10^{-5} \mathrm{~cm}^{2} \mathrm{~s}^{-1}$ and an oil-air partition coefficient of 1.6 (free energy of $-1.2 \mathrm{~kJ} \mathrm{~mol}^{-1}$ ) have been reported, resulting in a permeation resistance of $0.13 \times$ $10^{5} \mathrm{~s} \mathrm{~cm}^{-2}$. ${ }^{25}$ Considering the approximately 10-fold higher viscosity of wax esters compared to hexadecane, ${ }^{26,27}$ and the fact that diffusion is inversely proportional to viscosity, the simulation results appear to be in good agreement with experimental values. Moreover, considering the thickness range relevant for the TFLL $(10-100 \mathrm{~nm})$, a disordered BO film could hypothetically provide a total evaporation resistance of $0.2-2 \mathrm{~s} \mathrm{~cm}^{-1}$, assuming complete wetting.

Crystalline BO films with no defects had a complex evaporation resistance pattern with the highest resistance found in the region of the saturated alkoxy chains and resistance minima in the ester group and acyl chain regions. Average evaporation resistance was $1 \times 10^{16} \mathrm{~s} \mathrm{~cm}^{-2}$ (see Figure 3D), mainly because of the large free-energy barrier (Figure 3C). Diffusion coefficient within the crystalline film was also approximately one order of magnitude smaller compared to the disordered film, but it had only a minor effect on the evaporation resistance. The large free-energy barrier of the crystalline layer yields a total permeation resistance of $1 \times 10^{9}$ $\mathrm{s} \mathrm{cm}^{-1}$ for a single-crystalline $\mathrm{BO}$ layer, which is comparable to a $1 \mathrm{~mm}$ thick film of amorphous low-density polyethylene. ${ }^{28}$
However, as can be seen from Figure 3, defects in the crystalline layer can reduce the free-energy barrier, resulting in a lower total permeation resistance of approximately $20 \mathrm{~s} \mathrm{~cm}^{-1}$, comparable to that of monolayers of evaporation-resistant polar lipids, such as docosanol. ${ }^{4}$ It should be noted that the permeation resistance through a crystal defect depends on the exact nature of the defect, and the results presented here are therefore indicative, demonstrating increased water permeability in crystallite boundary regions. While determining the effect of different defects is beyond the scope of this study, the results shown in Figure 3 suggest that the evaporation resistance of a solid WE film is determined by the amount of lattice defects and grain boundaries, because permeation through an intact crystal is negligible. This is in line with previous works that have shown permeation of water ${ }^{29}$ and oxygen $^{30}$ through condensed lipid monolayers to occur mainly through interdomain boundaries and intradomain defects. In fact, even microscopic holes are common in solid BO films, persisting for tens of minutes (Figure S5).

The present study is the first one in which it has been shown in molecular detail how not only the lipid content but also the phase state and the structural organization of the TFLL critically determine its ability to minimize the evaporation of aqueous tear fluid from the ocular surface and therefore to avoid symptoms of DES. The central factors neglected in previous studies are wax esters in the crystalline state that in our atomistic simulations were observed to reduce evaporation of tear fluid in a concerted manner. Previous simulation studies ${ }^{31}$ have missed finding this critical piece of knowledge, and the reason is quite simple: all the previous simulations have been based on the use of coarse-grained models, ${ }^{32}$ which allow simulations of large systems at microsecond time scales but lack the ability to describe crystalline order. This is the key 
reason why the present work was conducted through atomistic simulations despite their considerable computational load.

Our findings have several important implications considering the structure and function of the TFLL. First, the TFLL-like WEs spread as thin films on a water surface as temperature approaches the melting point of the WE. Tear film WEs likely behave in a similar manner on the tear film surface, because the physiological temperature of the ocular surface matches the melting point determined for tear film lipids. ${ }^{33-35}$ The fact that pure WEs spread under these conditions on a water surface contradicts the view that polar lipids, such as O-acyl- $\omega$-hydroxy fatty acids or phospholipids, are required for the formation of a stable lipid film on the tear film surface. The limited importance of polar lipids would be consistent with the most recent lipidomic analyses, which suggest that the TFLL may contain only small amounts of polar lipids. ${ }^{5-7,36}$ Second, because the TFLL is partially crystalline, it is likely that crystallization takes place at the water-lipid interface, or in its vicinity, similarly to what was described for BO in this study. Further studies of multicomponent TFLLs would be highly welcome, with lipid content matching realistic TFLL compositions, ${ }^{5-7}$ and are currently being done. Third, reduction of evaporation is likely to result from organized crystalline layers within the TFLL at the water-lipid interface. Clinical studies have shown that TFLL thicknesses of only 10$20 \mathrm{~nm}$ suffice to produce normal tear film thinning rates. ${ }^{37,38}$ As shown above, a crystalline $\mathrm{BO}$ layer (thickness $\approx 5 \mathrm{~nm}$ ) with no defects could hypothetically reduce evaporation by close to $100 \%$. The most important parameters would then be the surface coverage and the number of defects and grain boundaries in the crystalline layer. Local defects could lead to faster local evaporation, which has recently been suggested as a mechanism for tear film breakup. ${ }^{39}$

To prevent evaporation from the tear film during the interblink period, such a film would need to reach a crystalline state and high coverage within seconds after the blink. Therefore, uncovering the critical parameters associated with the nonequilibrium spreading and crystallization of such interfacial films may reveal potential pathological mechanisms and new possibilities for the treatment of DES.

\section{ASSOCIATED CONTENT}

\section{(S) Supporting Information}

The Supporting Information is available free of charge on the ACS Publications website at DOI: 10.1021/acs.jpclett.9b01187.

Details of both computational and experimental methods and additional results (PDF)

Topology files in a GROMACS-compatible format (ZIP)

\section{AUTHOR INFORMATION}

\section{Corresponding Authors}

*E-mail: ilpo.vattulainen@helsinki.fi.

*E-mail: riku.o.paananen@helsinki.fi.

\section{ORCID $\odot$}

Matti Javanainen: 0000-0003-4858-364X

Ilpo Vattulainen: 0000-0001-7408-3214

\section{Notes}

The authors declare no competing financial interest.

IJ.M.H.: Deceased.

\section{ACKNOWLEDGMENTS}

We thank CSC-IT Center for Science (Espoo, Finland) for computing resources. R.O.P. thanks the Sigrid Juselius Foundation, Nissi Foundation, the Finnish Eye and Tissue Bank Foundation, the Jenny and Antti Wihuri Foundation, and the Orion Research Foundation for financial support. M.J. and I.V. acknowledge the Academy of Finland (Centre of Excellence program), the European Research Council (Advanced Grant CROWDED-PRO-LIPIDS), and the Sigrid Juselius Foundation for financial support. M.J. thanks Emil Aaltonen Foundation for financial support.

\section{REFERENCES}

(1) Stapleton, F.; Alves, M.; Bunya, V. Y.; Jalbert, I.; Lekhanont, K.; Malet, F.; Na, K.; Schaumberg, D.; Uchino, M.; Vehof, J.; Viso, E.; Vitale, S.; Jones, L. TFOS DEWS II Epidemiology Report. Ocul. Surf. 2017, 15, 334-365.

(2) Bron, A. J.; Tiffany, J. M.; Gouveia, S. M.; Yokoi, N.; Voon, L. W. Functional Aspects of the Tear Film Lipid Layer. Exp. Eye Res. 2004, 78, 347-360.

(3) Lemp, M. A.; Crews, L. A.; Bron, A. J.; Foulks, G. N.; Sullivan, B. D. Distribution of Aqueous-deficient and Evaporative Dry Eye in a Clinic-Based Patient Cohort: A Retrospective Study. Cornea 2012, 31, 472-478.

(4) Barnes, G. T. The Potential for Monolayers to Reduce the Evaporation of Water from Large Water Storages. Agric. Water Manage. 2008, 95, 339-353.

(5) Brown, S. H.; Kunnen, C. M.; Duchoslav, E.; Dolla, N. K.; Kelso, M. J.; Papas, E. B.; Lazon de la Jara, P.; Willcox, M. D.; Blanksby, S. J.; Mitchell, T. W. A Comparison of Patient Matched Meibum and Tear Lipidomes. Invest. Ophthalmol. Visual Sci. 2013, 54, 7417-7424.

(6) Lam, S. M.; Tong, L.; Duan, X.; Petznick, A.; Wenk, M. R.; Shui, G. Extensive Characterization of Human Tear Fluid Collected Using Different Techniques Unravels the Presence of Novel Lipid Amphiphiles. J. Lipid Res. 2014, 55, 289-298.

(7) Butovich, I. A. Tear Film Lipids. Exp. Eye Res. 2013, 117, 4-27.

(8) Rantamäki, A. H.; Javanainen, M.; Vattulainen, I.; Holopainen, J. M. Do Lipids Retard the Evaporation of the Tear Fluid? Invest. Ophthalmol. Visual Sci. 2012, 53, 6442-6447.

(9) Rantamäki, A. H.; Wiedmer, S. K.; Holopainen, J. M. Melting Points - The Key to the Anti-evaporative Effect of the Tear Film Wax Esters. Invest. Ophthalmol. Visual Sci. 2013, 54, 5211-5217.

(10) Paananen, R. O.; Rantamäki, A. H.; Holopainen, J. M. Antievaporative Mechanism of Wax Esters: Implications for the Function of Tear Fluid. Langmuir 2014, 30, 5897-5902.

(11) Leiske, D. L.; Miller, C. E.; Rosenfeld, L.; Cerretani, C.; Ayzner, A.; Lin, B.; Meron, M.; Senchyna, M.; Ketelson, H. A.; Meadows, D.; et al. Molecular Structure of Interfacial Human Meibum Films. Langmuir 2012, 28, 11858-11865.

(12) Rosenfeld, L.; Cerretani, C.; Leiske, D. L.; Toney, M. F.; Radke, C. J.; Fuller, G. G. Structural and Rheological Properties of Meibomian Lipid. Invest. Ophthalmol. Visual Sci. 2013, 54, 27202732.

(13) Sledge, S. M.; Khimji, H.; Borchman, D.; Oliver, A. L.; Michael, H.; Dennis, E. K.; Gerlach, D.; Bhola, R.; Stephen, E. Evaporation and Hydrocarbon Chain Conformation of Surface Lipid Films. Ocul. Surf. 2016, 14, 447-459.

(14) Lam, S. M.; Tong, L.; Reux, B.; Duan, X.; Petznick, A.; Yong, S. S.; Khee, C. B. S.; Lear, M. J.; Wenk, M. R.; Shui, G. Lipidomic Analysis of Human Tear Fluid Reveals Structure-specific Lipid Alterations in Dry Eye Syndrome. J. Lipid Res. 2014, 55, 299-306.

(15) Enkavi, G.; Javanainen, M.; Kulig, W.; Róg, T.; Vattulainen, I. Multiscale Simulations of Biological Membranes: The Challenge to Understand Biological Phenomena in a Living Substance. Chem. Rev. 2019, 119, 5607-5774.

(16) Jorgensen, W. L.; Maxwell, D. S.; Tirado-Rives, J. Development and Testing of the OPLS All-atom Force Field on Conformational 
Energetics and Properties of Organic Liquids. J. Am. Chem. Soc. 1996, $118,11225-11236$.

(17) Siu, S. W.; Pluhackova, K.; Böckmann, R. A. Optimization of the OPLS-AA Force Field for Long Hydrocarbons. J. Chem. Theory Comput. 2012, 8, 1459-1470.

(18) Izadi, S.; Anandakrishnan, R.; Onufriev, A. V. Building Water Models: A Different Approach. J. Phys. Chem. Lett. 2014, 5, 38633871.

(19) Javanainen, M.; Lamberg, A.; Cwiklik, L.; Vattulainen, I.; Ollila, O. H. S. Atomistic Model for Nearly Quantitative Simulations of Langmuir Monolayers. Langmuir 2018, 34, 2565-2572.

(20) Kohlhaas, R. Röntgenographische Untersuchung von definierten Einkristallen des Palmitinsäure-Cetylesters. Z. Kristallogr. Cryst. Mater. 1938, 98, 418-438.

(21) Dorset, D. L. Electronographic Crystal-structure Analysis of Cetyl Palmitate. Bioorg. Khim. 1976, 2, 781-788.

(22) Lutz, D. A.; Eddy, C. R.; Hunter, J. J. X-ray Diffraction Study of Some Normal Alkyl Esters of Long-Chain Acids. Lipids 1967, 2, 204207.

(23) Alexander, A. E.; Schulman, J. H. Orientation in Films of Longchain Esters. Proc. R. Soc. A 1937, 161, 115.

(24) Diamond, J. M.; Katz, Y. Interpretation of Nonelectrolyte Partition Coefficients Between Dimyristoyl Lecithin and Water. J. Membr. Biol. 1974, 17, 121-151.

(25) Schatzberg, P. Diffusion of Water Through Hydrocarbon Liquids. J. Polym. Sci., Part C: Polym. Symp. 1965, 10, 87-92.

(26) Aminabhavi, T. M.; Bindu, G. Densities, Viscosities, Refractive Indices, and Speeds of Sound of the Binary Mixtures of Bis(2methoxyethyl) Ether with Nonane, Decane, Dodecane, Tetradecane, and Hexadecane at 298.15, 308.15, and 318.15 K. J. Chem. Eng. Data 1994, 39, 529-534.

(27) Johnson, J. D.; Hinman, C. W. Oils and Rubber from Arid Land Plants. Science 1980, 208, 460-464.

(28) Börjesson, A.; Erdtman, E.; Ahlström, P.; Berlin, M.; Andersson, T.; Bolton, K. Molecular Modelling of Oxygen and Water Permeation in Polyethylene. Polymer 2013, 54, 2988-2998.

(29) Barnes, G. T. Permeation Through Monolayers. Colloids Surf., A 1997, 126, 149-158.

(30) Pu, G.; Longo, M. L.; Borden, M. A. Effect of Microstructure on Molecular Oxygen Permeation Through Condensed Phospholipid Monolayers. J. Am. Chem. Soc. 2005, 127, 6524-6525.

(31) Cwiklik, L. Tear Film Lipid Layer: A Molecular Level View. Biochim. Biophys. Acta, Biomembr. 2016, 1858, 2421-2430.

(32) Marrink, S. J.; Tieleman, D. P. Perspective on the Martini model. Chem. Soc. Rev. 2013, 42, 6801-6822.

(33) Leiske, D. L.; Leiske, C. I.; Leiske, D. R.; Toney, M. F.; Senchyna, M.; Ketelson, H. A.; Meadows, D. L.; Fuller, G. G. Temperature-Induced Transitions in the Structure and Interfacial Rheology of Human Meibum. Biophys. J. 2012, 102, 369-376.

(34) Butovich, I. A.; Lu, H.; McMahon, A.; Ketelson, H.; Senchyna, M.; Meadows, D.; Campbell, E.; Molai, M.; Linsenbardt, E. Biophysical and Morphological Evaluation of Human Normal and Dry Eye Meibum Using Hot Stage Polarized Light Microscopy. Invest. Ophthalmol. Visual Sci. 2014, 55, 87-101.

(35) Tan, J. H.; Ng, E. Y. K.; Acharya, U. R. Evaluation of Topographical Variation in Ocular Surface Temperature by Functional Infrared Thermography. Infrared Phys. Technol. 2011, 54, 469477.

(36) Chen, J.; Nichols, K. K.; Wilson, L.; Barnes, S.; Nichols, J. J. Untargeted Lipidomic Analysis of Human Tears: A New Approach for Quantification of O-acyl-omega Hydroxy Fatty Acids. Ocul. Surf. 2019, 17, 347-355.

(37) King-Smith, P. E.; Hinel, E. A.; Nichols, J. J. Application of a Novel Interferometric Method to Investigate the Relation between Lipid Layer Thickness and Tear Film Thinning. Invest. Ophthalmol. Visual Sci. 2010, 51, 2418-2423.

(38) Craig, J. P.; Tomlinson, A. Importance of the Lipid Layer in Human Tear Film Stability and Evaporation. Optom. Vis. Sci. 1997, 74, 8-13.
(39) Peng, C.; Cerretani, C.; Braun, R. J.; Radke, C. J. Evaporationdriven Instability of the Precorneal Tear Film. Adv. Colloid Interface Sci. 2014, 206, 250-264.

(40) Mills, R. Self-diffusion in Normal and Heavy Water in the Range 1-45 deg. J. Phys. Chem. 1973, 77, 685-688. 